\title{
Genetic components affecting embryonic developmental time of Drosophila melanogaster
}

\author{
Jurema Cruz do Nascimento ${ }^{1}$, Ivana Beatrice Mânica da Cruz ${ }^{1}$, Luiz Alberto Monjeló ${ }^{2}$ \\ and Alice Kalisz de Oliveira ${ }^{3}$ \\ ${ }^{1}$ Present address: Instituto de Biociências/Instituto de Geriatria, Pontificia Universidade Católica \\ do Rio Grande do Sul, Porto Alegre, RS, Brazil. \\ ${ }^{2}$ Depto. de Biologia, Instituto de Ciências Biológicas, Universidade Federal do Amazonas, Manaus, \\ AM, Brazil. \\ ${ }^{3}$ Depto. de Genética, Instituto de Biociências da Universidade Federal do Rio Grande do Sul, Porto Alegre, \\ RS, Brazil.
}

\begin{abstract}
The developmental time of the embryonic stage of Drosophila melanogaster was $21.66 \%$ faster and $14.75 \%$ slower than controls in populations selected for fast and slow developmental speed, respectively. The genetic model with two main loci with dominant and additive effect added to maternal effect and their relevant interactions can explain $96 \%$ of the phenotypic variability in the embryonic developmental time according to 14 crossing progenies involving fast and slow flies.
\end{abstract}

Key words: developmental rate, embryo, Drosophila

Received: April 5, 1999; accepted: June 7, 2002.

\section{Introduction}

The basic premise of insect development involves morphogenesis of quite different types of structures. The first is concerned with larval pattern building, and the second is metamorphosis-related to producing the adult body. These two different developmental processes are very related to embryonic stage, crucial in all pre-imaginal developmental fate, since it is responsible for further ontogenetic pathways. In this process, developmental time of each preimaginal stage, including embryonic period, also has two main roles: regulation of time needed for body development (genetic pattern), and fitting developmental time to environmental conditions (phenotype plasticity). This context makes pre-imaginal developmental time an adaptive trait and therefore a relevant selective component in insects living in unstable environments.

Several authors have considered this trait as only one event regulated by the same genetic pool during all insect stages (quantitative genes). The notion that Drosophila melanogaster in the wild has undergone continuous direc-

Send correspondence to Alice Kalisz de Oliveira. Departamento de Genética, Instituto de Biociências da Universidade Federal do Rio Grande do Sul, C.P. 15053, 91501-970 Porto Alegre, RS, Brazil. E-mail: alice.oliveira@ufrgs.br. tional selection for developmental speed has led some authors to suspect lack of additive genetic variance for the failure of many artificial attempts to produce faster developing lines (Sang and Clayton 1957, Clarke et al. 1961, Robertson 1963, Burnett et al. 1977, Partridge and Fowler 1992). Some studies analyzing embryonic developmental time in populations selected for few generations showed no differences in hatching time between developmental time selection treatments (Chippindale et al. 1994). Furthermore, selection for embryonic time produced very small differences, on the order of an hour or less between populations tested (Marinkovic and Ayala 1986, Neyfakh and Hartl 1994). Nevertheless, some authors have demonstrated that developmental rate is not strictly maximized in species like $D$. melanogaster although they have abundant and selectable genetic components for faster development (Zwann et al. 1995, Nunney 1996, Chippindale et al. 1997).

Divergent ideas of these authors probably relate to difficulties in isolating genetic and environmental contributions in each pre-imaginal developmental period. Probably, although a continuous directional selection exists in nature, this selective action is not necessarily continuous over all stages and, must therefore be analyzed in each development period. Due to the complex nature of the heritable developmental time pattern, a strong and continuous selection is 
needed to verify genetic components in each pre-imaginal stage. Here we analyzed the genetic contribution to embryo developmental time in crosses between $D$. melanogaster populations selected over more than 500 generations for developmental time extremes. The evolutionary role of embryonic directional selection is then discussed.

\section{Material and Methods}

The populations employed here descend from a single population of D. melanogaster, Oregon-R. Origin and subsequent maintenance of the populations were described by Oliveira and Cordeiro (1981). Briefly, the populations were mass-sampled and maintained as an outbred population during nearly two-week discrete generations under laboratory conditions : $24 \mathrm{~h}$ light, $25^{\circ} \mathrm{C}$ temperature, high relative humidity, and low to moderate larval and adult densities with abundant food.

The populations selected over 650 and 433 generations for short and long developmental time, were named $\mathrm{F}=$ fast population (short developmental period) and $\mathrm{S}=$ slow population (long developmental period).

Before selection the egg-adult cycle at $25^{\circ} \mathrm{C}$ was 10 days. At this temperature, after selection, the fast population adults start emerging on the seventh day after oviposition, whereas the slow population flies began emerging only on the $14^{\text {th }}$ day.

To select for fast development (F population) we bred the first flies that emerged, specially those of the eighth day. We let the flies age during two days and then we put them into eight $1 / 4 \mathrm{~L}$ capacity bottles with nearly 150 individuals that were replicated every three hours. The slow population selection (S) was similarly, but we bred the last flies that emerged, i.e., flies of the $13^{\text {th }}$ day or afterwards. After selecting this population, culture transfer was made every six hours, the time that maintains, at about the same level, competition in the more fertile fast population. The control population was maintained breeding all the flies ecloded between the eighth and $13^{\text {th }}$ days. Before replications the flies were mixed having population sizes consistently in the thousands of breeding adults to avoid confusing effects of inbreeding. Furthermore it is known that these populations posses abundant genetic variation (Oliveira and Cordeiro 1982 a, 1982 b, 1984; Loreto and Oliveira 1988).

The culture medium used is standard in our laboratory: $390 \mathrm{~g}$ coarse maize flour, $150 \mathrm{~g}$ full wheat flour, $310 \mathrm{~g}$ brown sugar, $20 \mathrm{~g}$ soybean flour, $10 \mathrm{~g}$ Nipagin, $3 \mathrm{~g}$ table salt, $2800 \mathrm{~mL}$ tap water.

Genetic analysis was performed on a population sample. To evaluate genetic additive, dominant, and maternal effects in the embryonic stage on developmental time it was recorded for all samples of parents $(\mathrm{P})$, first filial generation (F1), second filial generation (F2) and reciprocal backcrosses with the $\mathrm{S}$ and $\mathrm{F}$ flies. Ps and Pf were the slow and fast developmental parent generations respectively.
Parental populations were randomly divided into two groups for crosses and controls, kept for oviposition for one hour, and $15 \mathrm{~h}$ after oviposition the first instar emergence was counted. Statistical analysis was done using the SPSS computational program with the weighted least square multiple regression model to test the hypothesis of control of two main loci and gene interaction. The matrix used to isolate variation components was adapted from Mather and Jinks (1984) modified to include maternal effects (Oliveira and Cordeiro 1981) and corresponding interaction with genetic effects.

\section{Results}

Number of eggs examined, mean developmental time, and standard deviations in all progeny groups are shown in Table I.

Embryo developmental time ranged between $16 \mathrm{~h}$ (Pf) and $26 \mathrm{~h}(\mathrm{Ps})$; the descendents of reciprocal crosses were intermediary between populations selected from extremes of developmental time. We did not observe superposition of development time between Pf and Ps. The first population hatched after $16 \mathrm{~h}$ to $20 \mathrm{~h}$ and the last, $23 \mathrm{~h}$ to $26 \mathrm{~h}$.

From these results, the hypothesis of two main loci controlling embryo developmental time was confirmed $\left(\mathrm{R}^{2}=0.9722 ; \mathrm{R}_{\text {adj }}^{2}=0.9639, \mathrm{SE}=0.0431\right)$ using the inverse of the average variance as the weight in the weighted least squares multiple regression. An analysis of variance of the regression model with three degrees of freedom for regression and 10 degrees of freedom for residual variation showed a $\mathrm{F}=116.48617(\mathrm{p}<0.0000)($ Table II).

Table I - Developmental time in embryonic stage of overall groups in a mating experiment of Drosophila melanogaster with progeny number $(\mathrm{N})$, mean and standard deviation (in hours).

\begin{tabular}{lrc}
\hline Crossing & $\mathrm{N}$ & Mean $\pm \mathrm{sd}$ \\
\hline Ps & 176 & $24.9 \pm 2.0$ \\
Pf & 122 & $17.0 \pm 1.1$ \\
F 1-1 & 58 & $19.6 \pm 1.9$ \\
F 1-2 & 775 & $20.5 \pm 1.2$ \\
F 2-1 & 731 & $18.5 \pm 1.5$ \\
F 2-2 & 392 & $19.1 \pm 2.1$ \\
B 1-1 & 168 & $21.0 \pm 3.0$ \\
B 1-2 & 170 & $20.7 \pm 1.9$ \\
B 1-3 & 136 & $18.6 \pm 1.4$ \\
B 1-4 & 176 & $19.3 \pm 2.3$ \\
B 2-1 & 1170 & $19.5 \pm 1.5$ \\
B 2-2 & 1441 & $17.8 \pm 1.1$ \\
B 2-3 & 1442 & $19.5 \pm 1.0$ \\
B 2-4 & 1649 & $17.5 \pm 1.2$ \\
C * & 79 & $21.7 \pm 2.2$ \\
\hline
\end{tabular}

$\mathrm{C} *$ control population maintained without selection. 
Table II - Regression model analysis of variance of embryonic developmental time in a mating experiment of Drosophila melanogaster involving 14 crossing groups.

\begin{tabular}{lcccc}
\hline & $\begin{array}{c}\text { Degree } \\
\text { freedom }\end{array}$ & $\begin{array}{c}\text { Sum of } \\
\text { square }\end{array}$ & $\begin{array}{c}\text { Mean } \\
\text { square }\end{array}$ & $\mathrm{F}$ \\
\hline Regression & 03 & 0.64842 & 0.21614 & $116.48617^{* *}$ \\
Residual & 10 & 0.01856 & 0.00186 & \\
\hline
\end{tabular}

$* *(\mathrm{p}<0.001)$

From regression model analysis, a single additive effect (A), a dominant and maternal (Dmo) and an additive and maternal (Amo) effect were observed. All had significant interaction effects on embryo developmental time (Table III). In the analyzed populations we found dominant effects of fast development genes since backcrossing with Pf showed nearly $100 \%$ fast descendents. Maternal effect was observed because the number of descendents of backcrossings with slow females differed from that observed from backcrossings with slow males, suggesting an extra-nuclear effect.

The chi-square test of goodness of fit for the regression model where $\chi^{2}=0.0184(p>0.01)$ was not significant, indicating that differences between developmental times observed and expected according to a regression model for the 14 crossings are irrelevant. Therefore, the genetic model with two main loci, maternal effect and their relevant interactions can explain $96 \%$ of phenotypic variability in embryo developmental time according to the 14 crossing progenies involving Pf and Ps flies.

\section{Discussion}

Selection for faster or slower rates of development in D. melanogaster is often unsuccessful, although a response is usually obtained when the selection is sustained for multiple generations (Tigerstadt 1969). As suggested by King (1959), embryonic rates differ for different populations of flies, an observation confirmed in our populations where among selected (Pf and Ps) and non-selected (Pc) populations, Pf showed shortest development time, Ps showed the longest, and the control was intermediate. In backcrosses, descendents were intermediate. These results indicate that

Table III - Estimate and t-test for genic and maternal effect using a regression model assuming two main independent loci controlling the developmental time in the Drosophila melanogaster embryonic stage.

\begin{tabular}{lrrrr}
\hline & $\beta$ & SD $\beta$ & t-value & t-sign \\
\hline DMo & -10.00 & 0.9335 & $10.71^{* *}$ & 0.0000 \\
AMo & 2.74 & 0.4227 & $6.49^{* *}$ & 0.0001 \\
A & 2.62 & 0.3725 & $7.04^{*}$ & 0.0000 \\
Constant & 19.58 & 0.1348 & $145.18^{* *}$ & 0.0000 \\
\hline
\end{tabular}

$\mathrm{DMo}=$ Dominant-maternal interaction effect; $\mathrm{AMo}=$ Additive-maternal interaction effect; $\mathrm{A}=$ Additive effect. $* *(\mathrm{p}<0.01)$. genetic variation for developmental rate exists in the populations. Results of the present paper also show that the embryo stage can be important in shortening developmental time because fast population reached embryo stage $21.66 \%$ faster than control population, whereas slow population developed $14.75 \%$ slower. Fast population egg-adult developmental time for males was $18.14 \%$ and for females $16.05 \%$ faster than control whereas slow population for males was $39.38 \%$ and for females $43.06 \%$ slower than control population (Da Cruz et al. 1995).

Selection for faster development in D. melanogaster populations of Chippindale et al. (1997) also resulted in accelerated populations that differed significantly from their controls after 10 generations. The response continued, apparently unabated, until the last experimental assay at generation 125. In this generation the accelerated treatments completed egg- to- adult development $15-17 \%$ faster than their controls. An earlier study of postponed aging populations (Chippindale et al. 1994) showed no differences in hatching time between selection treatments differentiated for developmental time. Furthermore, Chippindale et al. (1997) assumed that little or none of the evolutionary response was due to modification of egg duration. However, as in embryo stage practically all cells of the future adult individual are committed and determined, developmental time differences observed in this stage may be both responsible for some developmental constraints and important for the so-called rapid development syndrome.

The results here obtained suggest that $96 \%$ of developmental time phenotypic variability in the embryo stage is explained by two main loci with dominant, additive and maternal interaction effects. Another important biological trait with additive effects is longevity; however, the effects of these genes are swamped by the large amount of nonadditive genetic effects and genotype environmental interactions (Clare and Luckinbill 1985, Luckinbill and Clare 1985, Curtsinger 1990).

In the populations analyzed, we found dominant genetic effects for fast development because backcrossing with Pf population showed nearly $100 \%$ fast descendents. Nevertheless, maternal effect was also observed because the number of descendents of backcrossings with slow females was different from that observed in backcrossing with slow males, suggesting an extra-nuclear effect.

In the present study, strong evidence suggests that in the embryo stage, besides two main loci affecting developmental time, a maternal effect, interacting with dominant and additive effects, enters significantly in accounting for variability. The interaction between maternal and additive effect could be explained by some gene product remaining in ovum cytoplasm as a feedback mechanism, while maternal and dominant effect interaction might explain the variation found in crossings between different parental populations. 


\section{Acknowledgements}

We are grateful to Conselho Nacional de Pesquisas $(\mathrm{CNPq})$ and Fundação de Amparo à Pesquisa do Rio Grande do Sul (FAPERGS) for fellowships and grants.

\section{References}

Burnett B, Sewell D, and Bos M (1977) Genetic analysis of larval feeding behaviour in Drosophila melanogaster. II Growth relations and competition between selected lines. Genet. Res. 30:149-161.

Chippindale AK, Hoang DT, Service PM, and Rose MR (1994) The evolution of development in Drosophila melanogaster selected for postponed senescence. Evolution 48:1880-1899.

Chippindale AK, Alipaz JA, Chen Hsiao-Wei and Rose MR (1997) Experimental evolution of accelerated development in Drosophila 1. Developmental speed and larval survival. Evolution 51:1536-1551.

Clare MJ and Luckinbill LS (1985) The effects of gene environment interaction on the expression of longevity. Heredity 55:19-29.

Clarke JM, Maynard Smith J, and Sondhi KC (1961) Asymmetrical response to selection for rate of development in Drosophila subobscura . Genet. Res. 2:70-81.

Curtsinger J (1990) Genetic and environmental components in variance of longevity in Drosophila males. Talk presented at $43^{\text {rd }}$ Annual Meeting of Gerontology Society of America, 18 November.

Da Cruz IBM, Nascimento JC, Callegari-Jacques SM and Oliveira AK (1995) Adult life span in Drosophila melanogaster populations selected for long and short developmental period. Rev. Brasil. Genet. 18:23-30.

King JC (1959) Differences between populations in embryonic developmental rates. Amer. Nat. 93:171-180.

Loreto ELS and Oliveira AK (1988) Selection of Drosophila melanogaster for extremes of developmental rate. I. Structural and regulatory genetic divergence. Rev. Brasil. Genet. 11:253-265.

Luckinbill LS and Clare MJ (1985) Selection for lifespan in Drosophila melanogaster. Heredity 55:9-18.
Marinkovic D and Ayala FJ (1986) Selection for different rate of embryonic development in Drosophila melanogaster and Drosophila simulans. Genetika 18:205-219.

Mather K and Jinks JL (1984) Introduction to biometrical genetics. Chapman and Hall, New Felter Lane, London.

Neyfakh AA and Hartl DL (1994) Genetic control of the rate of embryonic development: selection for faster development at elevated temperatures. Evolution 47:1625-1631.

Nunney L (1996) The response to selection for fast larval development in Drosophila melanogaster and its effect on adult weight: an example of a fitness trade-off. Evolution 50:1193-1204.

Oliveira AK, and Cordeiro AR (1981) Genetic control of developmental rate and a mother factor in Drosophila melanogaster. Rev Brasil. Biol. 41:635-644.

Oliveira AK and Cordeiro A R (1982a) Alkaline phosphatase in Drosophila melanogaster selected for extremes of developmental rate and a recessive allele. Rev. Brasil. Genet. 4:135-147.

Oliveira AK and Cordeiro AR (1982b) Genetics of Esterase A and its relationships with the developmental rate in selected strains of Drosophila melanogaster. Rev. Brasil. Biol. 42:133-140.

Oliveira AK and Cordeiro AR (1984) Ontogenetic changes in isoenzymes expression pattern of Drosophila melanogaster selected for high and slow developmental rate. Rev. Brasil. Biol. 44:133-140.

Partridge L and Fowler K (1992) Direct and correlated responses to selection on age at reproduction in Drosophila melanogaster. Evolution 46:76-91.

Robertson FW (1963) The ecological genetics of growth in Drosophila melanogaster. VI. The genetic correlation between the duration of the larval period and body size in relation to larval diet. Genet. Res. 4:74-92.

Sang JH and Clayton GA (1957) Selection for larval development time in Drosophila. J. Hered. 48:265-270.

Tigerstadt PMA (1969) Experiments on selection for developmental rate in Drosophila melanogaster. Ann. Acad. Sci. Fenn. Serr. A IV Biol. 148:1-53.

Zwaan BJ, Bijlsma R and Hoekstra RF (1995) Artificial selection for developmental time in Drosophila melanogaster in relation to the evolution of aging: direct and correlated responses. Evolution 49:635-648. 